\title{
Quantitative analysis of the relationship between microRNA-124a, $-34 b$ and -203 gene methylation and cervical oncogenesis
}

\author{
ANCA BOTEZATU $^{1}$, CRISTINA DANIELA GOIA-RUSANU ${ }^{1}$, IULIA VIRGINIA IANCU ${ }^{1}$, IRINA HUICA ${ }^{1}$, \\ ADRIANA PLESA $^{1}$, DEMETRA SOCOLOV ${ }^{2}$, CARMEN UNGUREANU ${ }^{3}$ and GABRIELA ANTON ${ }^{1}$ \\ ${ }^{1}$ Viral Genetic Engineering Laboratory, Romanian Academy 'Stefan S. Nicolau’ Virology Institute, 030304 Bucharest; \\ Departments of ${ }^{2}$ Obstetrics and Gynecology, and ${ }^{3}$ Pathology, 'Grigore T. Popa' \\ University of Medicine and Pharmacy, 700115 Iassy, Romania
}

Received August 11, 2010; Accepted October 21, 2010

DOI: $10.3892 / \mathrm{mmr} .2010 .394$

\begin{abstract}
Cervical cancer is a leading cause of mortality in women. Molecular and epidemiological data have unequivocally confirmed that high-risk human papillomaviruses (HPVs) are a major etiological agent of this malignancy, as host epigenetic alterations are induced in response to viral infection. The present study evaluated the methylation status of $\mathrm{CpG}$ islands surrounding $m i R-124 a$, $m i R-34 b$ and $m i R-203$ in 29 cervical cancer precursor lesions, 31 cervical tumors and 30 normal control samples, with the aim of identifying potential markers of cervical cancer. Direct quantitative methylation-specific PCR (qMSP) was used to evaluate the degree of methylation in the samples. HPV DNA was detected and genotyped using the Linear Array HPV Genotyping Test. Data were statistically analyzed using the Kruskal-Wallis test. Differences in miRNA hypermethylation between the tumor and control samples were highly significant for all the genes tested $(\mathrm{p}<0.0001)$. Significant results were also obtained regarding the hypermethylation of $m i R-124 a$ and $m i R-203$ in the precursor lesions compared to the control samples. Among the 29 patients with precursor lesions, 68.97\% (20/29) presented high risk (hr)-HPV genotypes and $31.03 \%(9 / 29)$ were diagnosed with low risk (lr)-HPV. Significant results $(\mathrm{p}=0.0266)$ were obtained for $m i R-124 a$ (hr-HPV group, mean 41.32; lr-HPV group, mean 6.74), revealing a strong association between the methylation process and the hr-HPV genotype. Borderline results $(\mathrm{p}=0.058)$ were obtained for $m i R-203$ (hr-HPV group, mean 44.05; lr-HPV group, mean 3.33). These results confirm the involvement of epigenetic alterations in cervical oncogenesis. The lr-HPV precursor lesions had a methylation percent pattern similar to that of the normal samples, while the results for the hr-HPV precursor lesions and tumors indicate a possible involvement of the hr-HPV genotype in the miRNA methylation process.
\end{abstract}

Correspondence to: Anca Botezatu, Viral Genetic Engineering Laboratory, Romanian Academy 'Stefan S. Nicolau' Virology Institute, 285 Mihai Bravu Ave., Bucharest 030304, Romania

E-mail: gnanka30@yahoo.com

Key words: miRNAs, human papillomavirus, cervical cancer, DNA methylation

\section{Introduction}

Cervical cancer is the third most common cancer overall and the leading cause of cancer-related mortality among women in developing countries. At least 370,000 new cases are identified each year worldwide, $80 \%$ of which are in developing countries (www.nccc-online.org). Romania has a higher incidence of cervical cancer than any other European country. In 2004 , the US had a 2.4 per 100,000 mortality rate due to cervical cancer; by contrast, the mortality rate in Romania was approximately 14.6 per $100,000(1,2)$. The main cause of cervical cancer is high-risk (hr)-human papillomavirus (HPV) infection (3). The impact of viral infection on cell function includes the deregulation of cell cycle control and metabolism, epigenetic alterations and genomic instability $(4,5)$.

The prevention and diagnosis of cervical cancer is based on cytological (Papanicolaou test) and hystopathological exams. Currently, women with abnormal cytology are tested for the presence of viral DNA and undergo genotyping. It is well known that not all women who present an hr-HPV genotype develop cervical cancer; therefore, it is important to identify new potential biomarkers for discerning women at risk of developing cervical cancer.

In addition to the major role of genetic modifications (mutations) in cancer development, epigenetic alterations are important and reversible events in the oncogenic process. Epigenetic factors are involved in several aspects of the regulation of genome physiology, including DNA methylation, histone modification and RNA interference.

In cancer cells, promoter hypermethylation of tumor suppressor genes induces the transcriptional repression of these genes, and has a major impact on the control of cell function (6). Non-coding micro RNAs (miRNAs), which are involved in the regulation of gene expression through mRNA degradation, may contribute to cancer development and progression. The post-transcriptional repression of miRNAs is a mechanism that suppresses the function of oncogenes or tumor suppressor genes; therefore, these small RNA molecules act as tumor or oncogene suppressors (7).

Certain miRNAs have been identified as overexpressed in various types of tumors. For example, miR-155 is overexpressed in chronic lymphocytic leukemia, B cell lymphoma, 
Hodgkin's and Burkitt's lymphoma, and breast cancer cells, indicating its oncogenic function $(8,9)$.

On the other hand, the expression levels of several miRNAs are down-regulated by promoter or surrounding $\mathrm{CpG}$ island hypermethylation. Recent studies using colon, breast and lung carcinoma, as well as leukemia and lymphoma cell lines, reported that $m i R-124 a$ frequently displayed this pattern, followed by the recruitment of methyl-CpG binding domain proteins. The down-regulation of $m i R-124 a$ expression in medulloblastomas induces the increased expression of its target, cyclin-dependent kinase 6 (CDK6). CDK6 interacts with cyclin $\mathrm{D}$ to form a complex involved in cell cycle progression (G1/S transition), and regulates the activity of $\mathrm{Rb}$ protein $(10,11)$. These reports suggest that $m i R-124 a$ has an important tumor suppressive role, and may contribute to the development of a malignant phenotype by altering the normal cell network control systems.

It has been reported that specific miRNA genes are downregulated in the presence of $\mathrm{CpG}$ island hypermethylation in oral squamous cell carcinomas, namely $m i R-34 b, m i R-137$, $m i R-193 a$ and miR-203 (12). Toyota et al demonstrated that $m i R-34 c / b$ are targets for $\mathrm{CpG}$ island hypermethylation in colorectal cancer (13). $m i R-34 b / c$ are known to regulate cell cycle proliferation by targeting components of the TP53 control pathway (14); therefore, it is not surprising that the $\mathrm{CpG}$ island hypermethylation of $m i R-34 \mathrm{~b} / \mathrm{c}$ together with that of other miRNAs (miR-148 and miR-9) is associated with the metastatic process (15).

The role of $m i R-203$ is controversial. Some authors have reported $m i R-203$ down-regulation in oral squamous cell carcinoma, while others found it to be up-regulated in ovarian cancer together with other miRNA genes (miR-21 and $m i R$-205) (16). The identification of tumor suppressor genes that undergo cancer-specific $\mathrm{CpG}$ island hypermethylation, and the correlation of these data with pre-neoplastic lesions, progression, tumor stage and long-term prognosis, are emerging fields of interest.

This study evaluated the methylation status of $\mathrm{CpG}$ islands surrounding miR-124a, $m i R-34 b$ and $m i R-203$ in cervical cancer and precursor lesions, and the relationship of this status with the presence of HPV DNA and the HPV genotype. To our knowledge, a substantial number of miRNAs are subject to epigenetic silencing in cancer, but only one study has investigated the epigenetic mechanisms (particularly methylation) that govern miRNA expression in cervical cancer (17).

\section{Materials and methods}

Sample collection. Biopsy tissues were obtained from 90 women with a median age of 33 years (range 21-65) who independently sought gynecological care at the Cuza Voda Gynaecology Clinic (Iasi, Romania). The patients were subdivided in three categories: i) the control group, which comprised 30 patients who underwent hysterectomy for reasons other than cervical lesions (mean age 30.66, range 21-56); ii) the precursor lesion group, comprising 29 patients with precursor cervical lesions (cervical intraepithelial neoplasia (CIN)I, II, III; mean age 32.65, range 21-60); iii) the tumor group, comprising 31 patients with cervical tumors (mean age 35.69, range 23-65) classified according to histological grade and
FIGO staging. No exclusion criteria (age, ethnicity) were applied. All biopsy samples were frozen at $-80^{\circ} \mathrm{C}$ for the isolation of nucleic acids. In order to eliminate false positive methylation results from the tissues, blood samples $(n=29)$ were collected from healthy volunteers. All the participants provided their informed concent, and the study protocol was approved by the Ethics Committee of the 'Grigore T. Popa' University of Medicine and Pharmacy.

DNA extraction. DNA extraction was performed using the High Pure PCR Template Preparation kit (Roche Molecular Biochemicals, Mannheim, Germany). The amount of each sample was established according to the manufacturer's recommendations. Whole blood $(300 \mu \mathrm{l}), 35 \mathrm{mg}$ of solid tumor tissue and cultured HeLa cells (10\%/culture) were used for DNA isolation. DNA samples were stored at $-20^{\circ} \mathrm{C}$. The concentration and purity of the DNA were evaluated using a NanoDrop spectrophotometer (NanoDropTechnologies, Montchanin, DE, USA). Total DNA isolated from the HeLa cell line was used to validate the results of methylation specific-PCR (MS-PCR) for the investigated miRNAs.

$H P V$ genotyping. HPV genotyping was performed using the Linear Array HPV genotyping test (Roche) according to the manufacturer's instructions. This test uses biotinylated primers to define a sequence of nucleotides within the polymorphic L1 region of the HPV genome of approximately $450 \mathrm{bp}$. A pool of HPV primers present in the Master Mix is designed to amplify HPV DNA from $37 \mathrm{HPV}$ genotypes, including 13 high risk types $(16,18,31,33,35,39,45,51,52,56,58,59$ and 68$)$. An additional primer pair targets the human $\beta$-globin gene as a control for cell adequacy, extraction and amplification.

Unmethylated $C$ residue conversion. Unmethylated $\mathrm{C}$ residue conversion was performed with the EpiTect Bisulfite kit (Qiagen, Valencia, California, USA). Incubation of the target DNA with sodium bisulphite results in the conversion of unmethylated cytosine residues into uracil, leaving the methylated cytosines unchanged. Isolated DNA obtained from biopsies, tumors, HeLa cells and blood (700 $\mathrm{ng} / \mu \mathrm{l}$ from each) were subjected to bisulphite treatment, along with positive/negative controls (CpGenome Universal Methylated DNA/CpGenome Universal Unmethylated DNA, Millipore, Billerica, MA, USA). Aliquots of the treated samples were stored at $-80^{\circ} \mathrm{C}$.

Primers. Primers were designed using the online bioinformatics tool MethPrimer (www.urogene.org/methprimer) to discriminate between the methylated and unmethylated status of the investigated miRNA genes. The primers were selected and tested in silico in order to avoid non-specific alignments. MethPrimer converts the DNA sequence into a bisulphitetreated DNA sequence, and displays the density of $\mathrm{CpG}$ islands. The specific DNA sequences for the targeted miRNAs were selected from the NCBI database and were synthesized by Invitrogen Corp. (Carlsbad, CA, USA).

Methylation specific-PCR. MS-PCR consists of two different PCRs that amplify a bisulphite-treated DNA sample with primers for methylated and unmethylated sequences. MS-PCR 


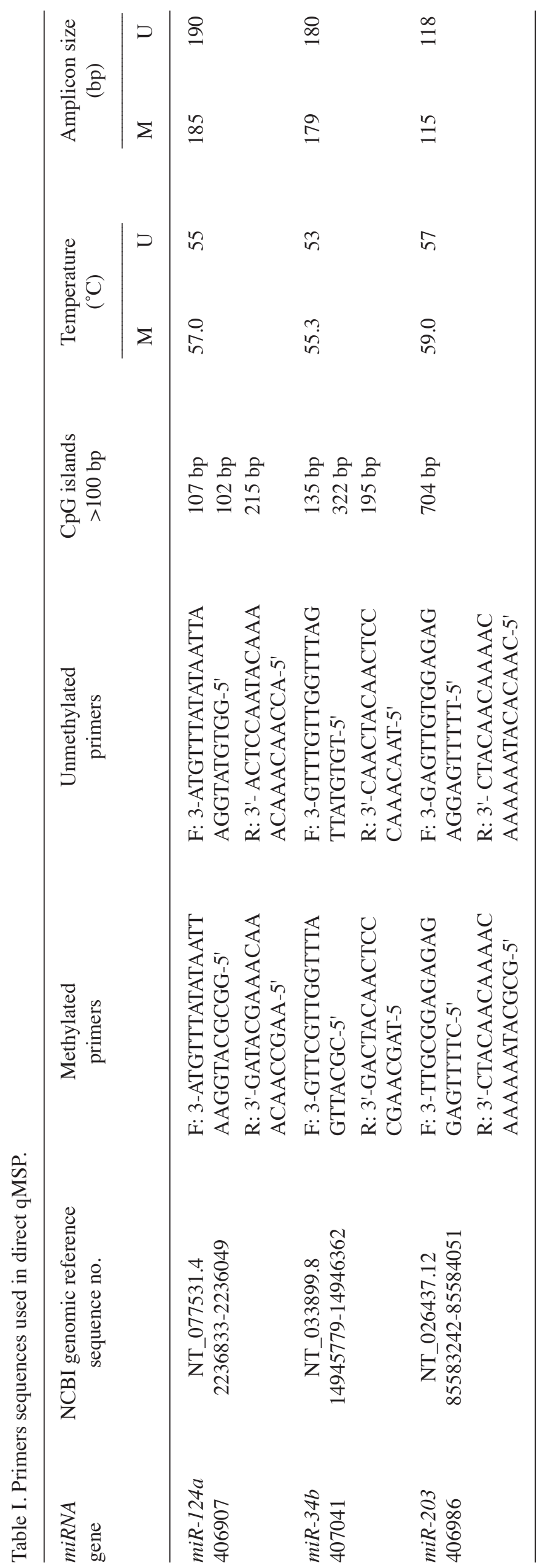

was performed using Platinum Taq DNA Polymerase (1U), $1 \mathrm{X}$ enzyme buffer, $1.5 \mathrm{mM} \mathrm{MgCl}_{2}, 200 \mu \mathrm{M}$ of each dNTP, $0.3 \mu \mathrm{M}$ of each specific primer and $5 \mu \mathrm{l}$ of target DNA, in a final volume of $25 \mu \mathrm{l}$. The PCR conditions are shown in Table I. The amplicons were evaluated on $2 \%$ agarose electrophoresis gel. The presence or absence of one of the amplicons was correlated with the pattern of the target gene.

Direct qMSP of genomic DNA. Direct quantitative methylationspecific PCR (qMSP) of genomic DNA, a quantitative method for evaluating the degree of methylation, was conducted using the 7300/7500/7500 Fast Real-Time PCR System (Applied Biosystems, Foster City, CA, USA). Standard curves were generated using positive (fully methylated) and negative (fully unmethylated) controls serially diluted to $10 \mathrm{pg}, 100 \mathrm{pg}$, $1 \mathrm{ng}$ and $10 \mathrm{ng}$ according to the manufacturer's instructions. DNA samples were diluted to a final concentration of $10 \mathrm{ng} /$ $\mu 1$. FastStart Universal SYBR Green Master (ROX) $(25 \mu \mathrm{l})$ (Roche), $0.3 \mu \mathrm{M}$ primers and $5 \mu \mathrm{l}$ DNA were used. Direct qMSP was performed in a 50- $\mu 1$ final volume for an initial cycle of $10 \mathrm{~min}$ at $95^{\circ} \mathrm{C}$, followed by 40 cycles of $15 \mathrm{sec}$ at $95^{\circ} \mathrm{C}$ and $60 \mathrm{sec}$ at the annealing temperatures specified in Table II.

qPCR experiments were performed in duplicate $(\Delta \mathrm{Ct} \approx 1.2$ between replicates) and mean values were used for the calculations. In order to establish the quality of the DNA samples, the $A C T B$ gene was used as a reference (17). All samples considered negative for methylation presented a $\mathrm{Ct}$ for $A C T B$ below 35 , excluding false negative results.

Methylation percent calculation. The methylation percent (\%M) was calculated according to the method of Fackler et al (18). The concentration of unmethylated (U) and methylated (M) DNA for each patient sample was extrapolated using the standard curves. The percentage of methylation was calculated according to the formula: $\% \mathrm{M}=100 \times[\mathrm{ng} \mathrm{M}$ gene $\mathrm{A} /(\mathrm{ng} \mathrm{M}$ gene $\mathrm{A}+\mathrm{U}$ gene $\mathrm{A})]$, where the total target gene DNA was taken as the sum of $\mathrm{U}+\mathrm{M}$.

Statistical analysis. Statistical analysis was performed with GraphPad InStat3 (GraphPad Software Inc., San Diego, CA, USA). The Kruskal-Wallis test was used to determine whether the groups had the same medians or the same distribution. p-values $<0.05$ were considered significant. For contingency table analysis, Fisher's exact test was used. Odds ratios, relative risk, specificity and sensitivity were calculated. Fisher's exact test provided the positive predicting value (PPV), which represents the probability of a patient positive for target gene methylation to have cervical cancer.

\section{Results}

Qualitative $M S-P C R$. After bisulphite treatment of the samples, MS-PCR was performed in order to evaluate the methylation status of the HeLa cell line and to validate the ability of the primers to discriminate between methylated and unmethylated status. For this purpose, DNA controls for total methylated and unmethylated sequences, as well as DNA from tumor samples, were used. The optimal PCR temperatures for each primer were established. The results are shown in Fig. 1. 
Table II. MS-PCR conditions for each investigated miRNA gene.

\begin{tabular}{clc}
\hline Time & Temperature $\left({ }^{\circ} \mathrm{C}\right)$ & No. of cycles \\
\hline $5 \mathrm{~min}$ & 94 & 1 \\
$30 \mathrm{sec}$ & 94 & 35 \\
$30 \mathrm{sec}$ & $56(m i R-124 a)$ & 35 \\
$30 \mathrm{sec}$ & $54(m i R-34 b)$ & 35 \\
$30 \mathrm{sec}$ & $58(m i R-203)$ & 35 \\
$30 \mathrm{sec}$ & 72 & 35 \\
$7 \mathrm{~min}$ & 72 & 1 \\
\hline
\end{tabular}

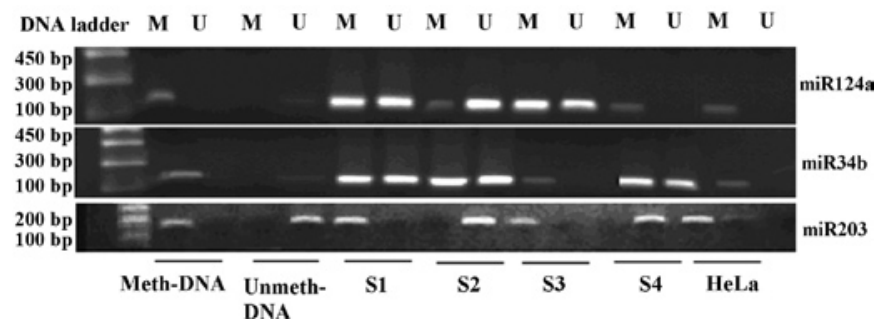

Figure 1. Agarose gel electrophoresis of methylated (M) and unmethylated (U) DNA controls, HeLa cells and 4 representative tumor samples (S1-S4). Top panel: amplicons detected for miR124a controls and samples, $185 \mathrm{bp}-\mathrm{M}$ and $190 \mathrm{bp}-\mathrm{U}$; middle panel: amplicons for $m i R 34 b, 179 \mathrm{bp}-\mathrm{M}$ and $180 \mathrm{bp}-\mathrm{U}$; bottom panel: amplicons for miR203, $115 \mathrm{bp}-\mathrm{M}$ and $118 \mathrm{bp}-\mathrm{U}$.
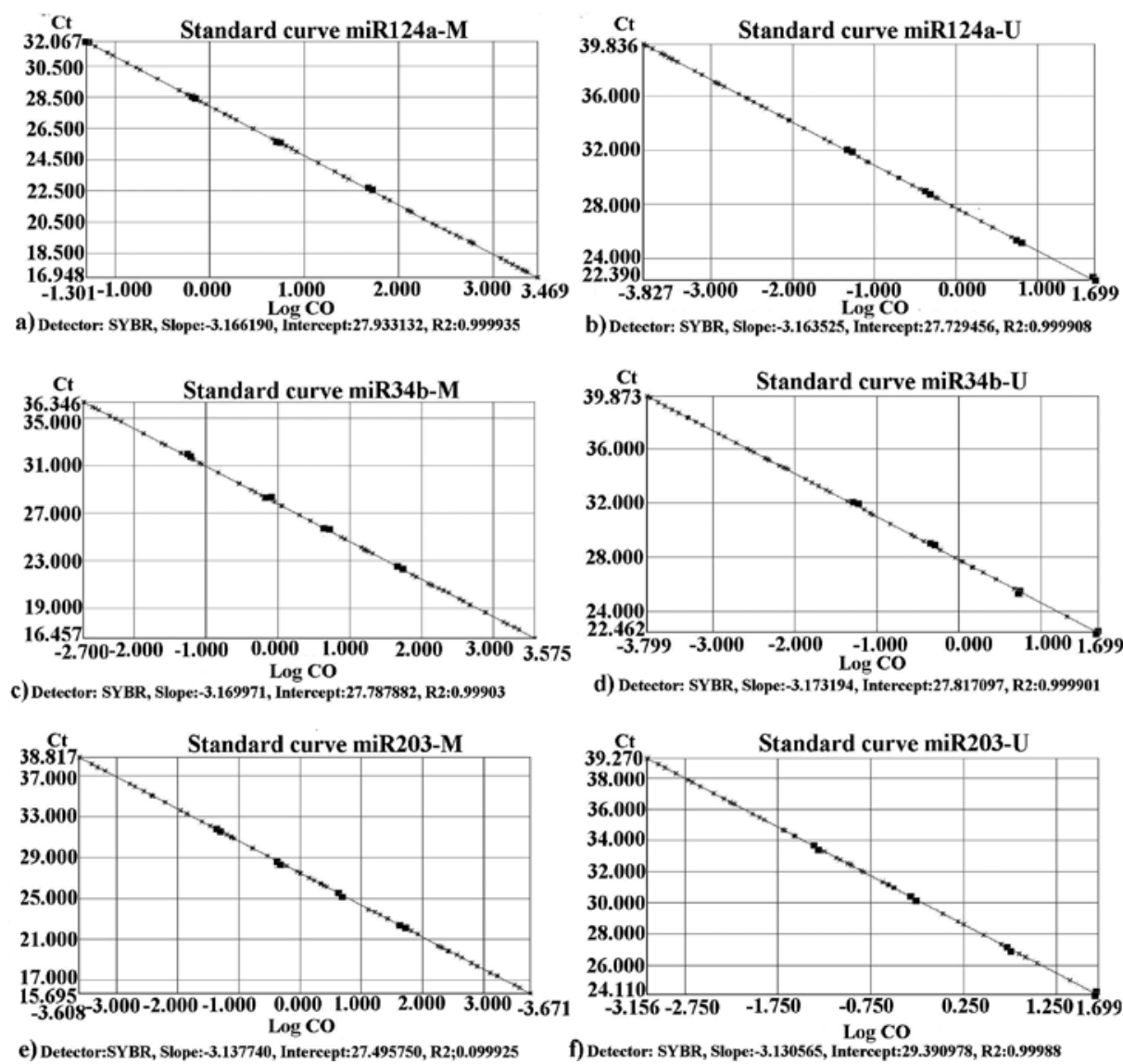

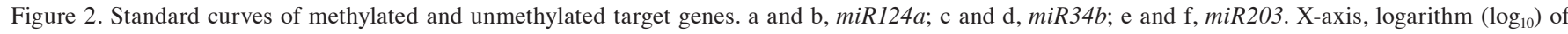
sample concentration; Y-axis, $\mathrm{Ct}$ value.

Validation of direct $q M S P$. Primer performance was evaluated for specific amplification of methylated and unmethylated DNA in the qPCR reaction. The $\triangle \mathrm{Ct}=\mathrm{CtM}-\mathrm{CtU}$ formula was calculated as a function of sample dilution over the range specified for the specific standards. The differences between $\Delta \mathrm{Ct}$ values were similar for all dilutions $(\Delta \mathrm{Ct} m i R-124 a \approx$ $0.726 ; \Delta \mathrm{Ct} m i R-34 b \approx 0.575, \Delta \mathrm{Ct} m i R-203 \approx 2.8930$ ). On the other hand, the slope value for all the standard curves was around -3.16, indicating a 2 -fold increase in PCR product per cycle during the linear phase of real-time PCR. The average of the calculated efficiencies was $\sim 107.3$. Fig. 2 shows that the correlation coefficient $(R 2)$ was 0.999 , providing evidence of linearity over the entire range of template concentrations.
Quantification of methylation in squamous cell carcinoma and cervical precursor lesions. The unmethylated standards were negative for methylation and yielded a $\% \mathrm{M}$ value of $\sim 0$. The methylated standards revealed no amplification using unmethylated primers. In order to eliminate false positive $\% \mathrm{M}$ values due to blood cell infiltration into tissue, a cutoff was established according to the percentage of methylation for each miRNA in the blood samples. The analysis of $\% \mathrm{M}$ in the blood samples revealed that this event occurred rarely; therefore, all the samples presenting a $\% \mathrm{M}$ under the cutoff were considered negative. The cutoffs were calculated using the 95 th percentile from blood $\% \mathrm{M}$ values $(95 \%$ of the blood samples were at or below the cutoffs). The obtained cutoff 
Table III. Direct qMSP analysis.

\begin{tabular}{|c|c|c|c|c|c|c|}
\hline $\begin{array}{l}\operatorname{miRNA} \\
\text { gene }\end{array}$ & Range & Median & Mean $\pm \mathrm{SE}$ & $\begin{array}{c}\text { Lower } 95 \% \\
\text { confidence limit }\end{array}$ & $\begin{array}{c}\text { Upper } 95 \% \\
\text { confidence limit }\end{array}$ & P-value \\
\hline \multicolumn{7}{|l|}{ Normal } \\
\hline$m i R-124 a$ & $0-7.33$ & 0.0 & $0.359 \pm 0.260$ & -1.663 & 0.8863 & $<0.0001$ \\
\hline$m i R-34 b$ & $0-86.5$ & 0.0 & $4.382 \pm 3.028$ & -1.810 & 10.573 & $<0.0001$ \\
\hline$m i R-203$ & $0-44.9$ & 0.0 & $2.300 \pm 1.646$ & -1.069 & 5.664 & $<0.0001$ \\
\hline \multicolumn{7}{|c|}{ Precursor lesions } \\
\hline$m i R-124 a$ & $0-100$ & 0.0 & $27.49 \pm 6.700$ & 13.775 & 41.197 & $<0.050^{\mathrm{a}}$ \\
\hline$m i R-34 b$ & $0-100$ & 0.0 & $29.96 \pm 8.472$ & 12.609 & 47.308 & $>0.050^{\mathrm{a}}$ \\
\hline$m i R-203$ & $0-100$ & 0.0 & $33.14 \pm 8.007$ & 16.596 & 49.680 & $<0.050^{\mathrm{a}}$ \\
\hline \multicolumn{7}{|l|}{ Tumors } \\
\hline miR-124a & $0-100$ & 58.3 & $58.99 \pm 6.800$ & 45.119 & 72.880 & $<0.001^{\mathrm{a}}$ \\
\hline$m i R-34 b$ & $0-100$ & 43.2 & $47.54 \pm 8.645$ & 29.891 & 65.197 & $<0.001^{\mathrm{a}}$ \\
\hline$m i R-203$ & $0-100$ & 6.0 & $56.70 \pm 6.269$ & 43.896 & 69.499 & $<0.001^{\mathrm{a}}$ \\
\hline
\end{tabular}

${ }^{a}$ Compared to normal.

values were $7.206 \%$ for $m i R-124 a, 0.519 \% m i R-34 b$ and $2.099 \%$ for $m i R-203$. Some samples presented methylation levels below the cutoff (Fig. 3).

All three miRNA genes were hypermethylated in the precursor lesions and tumor samples (Tables III and IV). CpG island methylation of the miR-124a gene was confirmed in $13.33 \%(4 / 30)$ of the normal control samples, compared to $41.38 \%(12 / 29)$ of the precursor lesions and $90.33 \%(28 / 31)$ of the tumor samples. Hypermethylation of the $m i R-34 b$ gene was found in 2/30 (6.67\%) of the control samples, compared to 9/29 (31.03\%) of the precursor lesions and 23/31 (74.19\%) of the tumor samples. Among the control cases, 10\% (3/30) of the samples were positive for $m i R-203$ gene hypermethylation, compared to $11 / 29(37.93 \%)$ of the precursor lesions and $27 / 31(87.09 \%)$ of the tumor samples. The methylation range of the investigated miRNA in each patient group is presented in Table II.

When the Kruskal-Wallis test was applied, the differences between the group medians were highly significant for all the tested genes $(\mathrm{p}<0.0001)$. Significant results were obtained regarding the hypermethylation values of the precursor lesion group compared to the control group for the miR-124a and miR-203 genes (Table III). The hypermethylation percentage values for the HeLa cell line were also high: $80 \%$ for $m i R-124 a, 75 \%$ for $m i R-34 b$ and $65 \%$ for $m i R-203$.

The analysis of patient and control group blood samples revealed low or undetectable methylation. Median leukocyte methylation was $0.58 \%$ for $m i R-124 a, 0.09 \%$ for $m i R-34 b$ and $0.61 \%$ for $m i R-203$. Therefore, we concluded that the methylation percent observed in tumor and precursor lesions (Fig. 3) is derived largely from abnormal cells, rather than from normal cells surrounding the precursor/tumor lesions and/or infiltrating leukocytes. No significant differences were observed between different stages among carcinoma patients, with the exception of the $m i R-124 a$ gene, which appears to have an increasing $\% \mathrm{M}$ pattern, from carcinoma in situ (CIS) to stage IVA (Fig. 4).

The mean differences between the stages were not significant $(\mathrm{p}>0.05)$. However, although it was not possible to discriminate between miRNA hypermethylation at the various

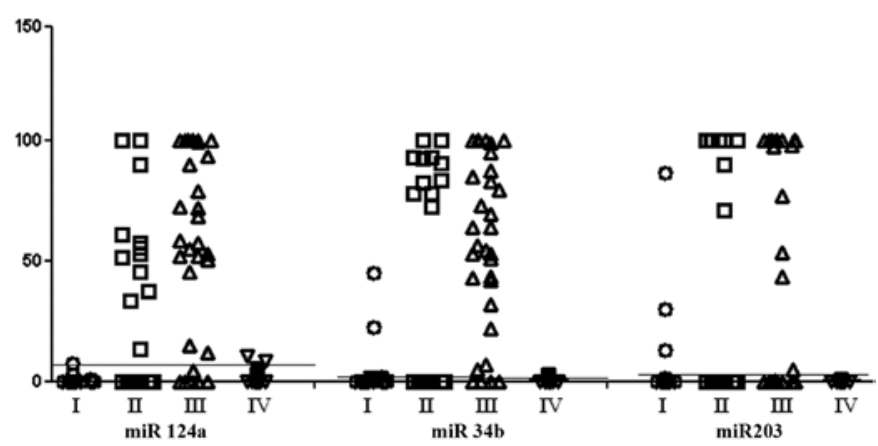

Figure 3. Grouped column scatter representation of case distribution vs $m i R N A$ gene hypermethylation. Horizontal lines represent the cutoffs.

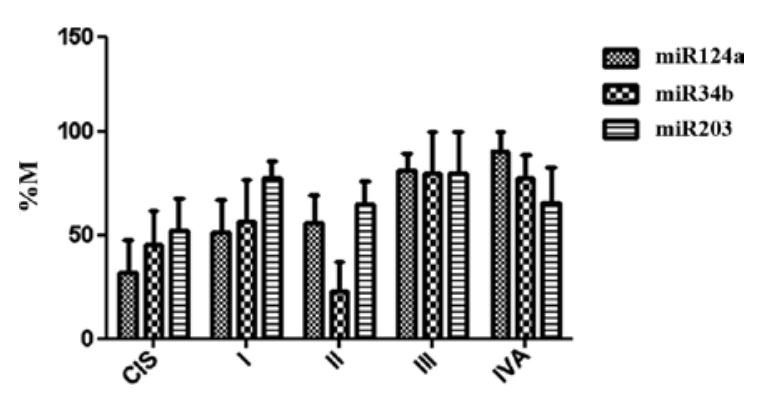

Figure 4. \% M means for target miRNA genes in tumors of different stages: carcinoma in situ (CIS), I, II, III, IVA. Data represent the mean \pm SD.

stages of carcinogenesis, it is likely that this process is linked to oncogenesis.

HPV DNA detection and genotyping. No HPV DNA was detected in the control group. In the group of patients with precursor lesions, 69\% (20/29) were found to be hrHPVpositive and $31 \%(9 / 29)$ were lrHPV-positive. hrHPVs types $16,18,33$ and 58 were most frequently detected. In the group with cervical tumors, $9.3 \%$ of the cases were HPV-negative; the HPV-positive patients presented only hrHPV types 16 and 18 . 


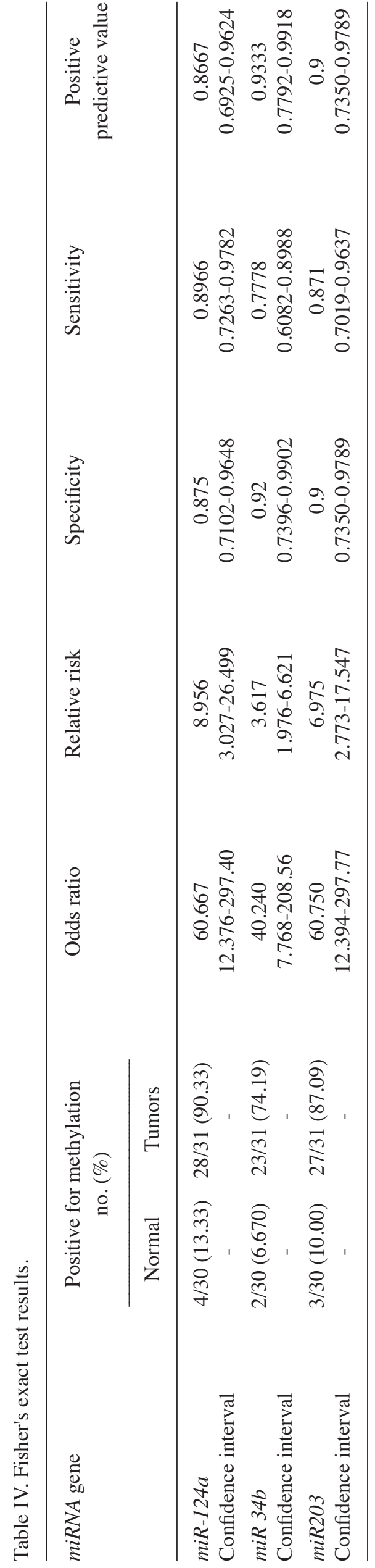

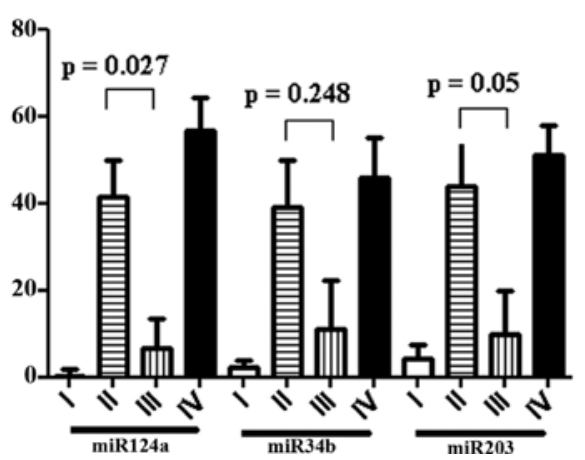

Figure 5. Methylation percent for target miRNA genes and association with the presence of the lrHPV and hrHPV genotypes. I, normal (control) group; II, hr-HPV precursor lesion group; III, lr-HPV precursor lesion group; IV, hr-HPV tumor group. The p-values for the lr-HPV and hr-HPV positive patients are indicated. Data represent the mean \pm SEM.

The \% $\mathrm{M}$ values of the precursor lesion hr-HPV group were compared to those of the precursor lesion lr-HPV, tumor hr-HPV and control groups. In the precursor lesion group, significant results $(\mathrm{p}=0.0266)$ were obtained for $m i R-124 a$ (hr-HPV group, mean 41.32; lr-HPV group, mean 6.74), revealing a strong association between methylation and the hr-HPV genotype. Borderline results $(\mathrm{p}=0.058)$ were obtained for $m i R-203$ (hr-HPV group, mean 44.05; lr-HPV group, mean 3.33). No significant results $(\mathrm{p}=0.25)$ were obtained for $m i R-34 b(\mathrm{hr}-\mathrm{HPV}$ group, mean 38.98; lr-HPV group, mean 11.11).

The precursor lr-HPV lesion \%M values appeared to be similar to those of the control group, as no significant differences were observed in any of the investigated miRNAs. \%M values for hr-HPV precursor lesions did not differ significantly compared to the tumor group. On the other hand, the $\% \mathrm{M}$ of the hr-HPV precursor lesion group differed significantly from the control group, with the exception of $m i R-34 b$ (Fig. 5).

Specificity and sensibility of the miRNA methylation assays. Data regarding target gene hypermethylation of the control vs. tumor groups were analyzed using Fisher's exact test. Specificity (percentage of healthy patients identified as not having the condition) and sensitivity (prediction of the capacity to identify all patients from the tumor group presenting the disease) were calculated. The specificity and sensitivity were higher for $m i R-124 a$ (specificity $87.5 \%$, sensitivity $89.7 \%$ ) and $m i R-203$ (specificity $90 \%$, sensitivity $87.1 \%$ ), while for $m i R-34 b$, the sensitivity was slightly lower (specificity $92 \%$, sensitivity $77.8 \%$ ) (Table IV).

The odds ratios obtained indicated that the methylation of the miRNAs differentially increased the risk of cervical cancer development (60.67-fold for $m i R-124 a, 40.24$-fold for $m i R-34 b$ and 60.75-fold for $m i R$-203) compared to patients lacking the risk factor.

\section{Discussion}

miRNAs are involved in a wide range of biological processes through their post-transcriptional regulatory effects on mRNAs. Abnormal expression of miRNAs has been linked to the occurrence of various diseases, including immune system deregulation, cardiac dysfunction, neurodegenerative diseases 
and cancer. miRNAs that play a role in cancer development have been termed 'oncomirs', and appear to have a dual nature: some studies have indicated that oncomir expression is deregulated by the DNA methylation process (19), while others have confirmed their oncogenic role. It is therefore possible that future epigenetic findings will reveal a new code: the miRNA code.

There are studies linking the expression of specific miRNAs with specific cancers, but few have attempted to clarify the mechanisms of miRNA deregulation. The present study aimed to elucidate miRNA gene epigenetic regulation (DNA methylation). Given the high density of $\mathrm{CpG}$ islands present at the level of $m i R-124 a,-34 b$ and -203 , we hypothesized that there might be a good substrate for the DNA methylation process We assumed that, due to their tumor suppressor role, epigenetic silencing by methylation might support the involvement of miRNAs in the oncogenic process. Some studies have demonstrated that $m i R-124 a$ is hypermethylated in abnormal cells, whereas $m i R-127$ is methylated in both normal tissues and tumors $(20,21)$. Lujambio et al observed that the epigenetic silencing of $m i R-124 a$ in colon cancer cell lines resulted in the activation of CDK6 (15). By contrast, Hackanson et al targeted a new tumor suppressor gene, $C / E B P \alpha$, in acute myeloid leukemia, and found that $\mathrm{C} / \mathrm{EBP} \alpha$ protein levels were affected by the presence of $m i R-124 a(22)$.

Although the epigenetic silencing of tumor suppressor genes is an important feature of carcinogenesis, the epigenetic silencing of miRNAs remains unclear. Toyota et al discovered that the $m i R-34 b / c \mathrm{CpG}$ island is a bidirectional promoter, and that its methylation is associated with the silencing of $m i R-34 b / c$ and B-cell translocation gene 4 (BTG-4), a tumor suppressor gene (13).

Only a few studies have evaluated the expression levels of specific miRNAs in relation to their tumor suppressor role in cervical oncogenesis. In these studies, the expression level of $m i R-214$ was found to be down-regulated in cervical cancer compared to normal tissue (23), while pri-miR-34a expression was significantly reduced in cervical intraepithelial neoplasia and cervical cancer compared to normal cervical epithelium (24). Regarding the epigenetic substrate of $m i R N A$ regulation, in a very recent study, Wilting et al revealed that the $m i R-124$ family is hypermethylated to a high degree in the CaSki, SiHa and HeLa cell lines, and also in high-grade cervical lesions (17).

Our data confirmed that the methylation percent in the squamous cell carcinoma vs. the control group was statistically significant for all the investigated miRNAs. With the exception of $m i R-124 a$, no differences between the various stages of carcinogenesis and methylation percent were observed. A progressive methylation of this small molecule was observed from CIS to the IVA stage. When analyzing the methylation percent of the cervical precursor lesion group vs. the control group, significant results $(\mathrm{p}<0.05)$ were found only for $m i R-124 a$ and $m i R-203$. In an analysis of 139 cervical tissue specimens, Wilting et al revealed an increasing methylation frequency, from $0 \%$ in normal tissues up to $93 \%$ in cervical carcinomas, for $m i R-124-1$ and $m i R-124-2$, which is in agreement with our results for the same target.

An ideal tumor marker should allow for the screening, follow-up and determination of the best treatment option. It should be sensitive and specific, reflect the tumor grade and accurately predict outcome. Our study reveals that over $74 \%$ of cervical carcinomas presented hypermethylation of the investigated miRNA genes. The high frequency of hypermethylation, specificity and sensibility (range 0.871-0.92) indicates that the methylation status of the $124 a, 34 b$ and 230 miRNA genes may serve as a useful marker of cervical oncogenesis. The positive predictive value was high for all the miRNAs, indicating that almost all patients with positive results were correctly diagnosed. Although no significant statistical data regarding $m i R N A$ hypermethylation and cervical cancer stage were found, strong evidence links the hypermethylation process of the studied miRNA genes and cervical carcinogenesis. We found that the calculated relative risk value was higher than 1 for all the hypermethylated genes investigated (Table IV). This reveals a good correlation between the epigenetic silencing of tumor suppressor miRNAs and the occurrence of cervical cancer.

To the best of our knowledge, this is the first study concerning $m i R-230$ and $m i R-34 b$ hypermethylation in cervical cancer; only one of the miRNAs currently studied, $m i R-124 a$, was previously investigated by Wilting et al (17).

The lr-HPV precursor lesion group had a methylation percent pattern similar to that of the normal samples, while the methylation percent patterns of the hr-HPV precursor lesion and tumor groups were similar, indicating a possible involvement of the hr-HPV genotype in the regulation of miRNA gene expression. The association between the hr-HPV genotype and $m i R-34 b$ gene methylation has not been established. Our results are in contrast to those of Wilting et al (17), who did not observe any correlation between miR-124a methylation and the presence of the hr-HPV genotype. Wilting et al did correlate $m i R-124 a$ gene methylation with the cell immortalisation process; however, hr-HPV involvement in this process has already been established. In a very recent study, Melar-New and Laimins (25) revealed that $m i R-203$ expression levels were reduced by approximately 5-fold in HPV-positive cells. High levels of $m i R-203$ are inhibitory to HPV amplification. On the other hand, HPV proteins act as suppressors of $m i R-203$ gene expression in order to allow productive replication in differentiating cells. The link between hr-HPV genotypes and $m i R-203$ methylation percent established by our study may offer an explanation regarding the mechanisms of the downregulation of $m i R-203$ gene expression.

A significant number of patients from the hr-HPV precursor lesion group presented a high methylation percent (60\% for $m i R-124 a$ and $50 \%$ for $m i R-203$ ). The hr-HPV genotype positive patients from the precursor lesions group with a high methylation percent were more likely to develop cervical cancer. Therefore, the detection of methylation percent may be a useful tool for the triage of hr-HPV-positive patients with abnormal cytology.

This article provides novel findings regarding the methylation pattern of miRNAs in cervical oncogenesis. Our results are accompanied by discoveries in this field regarding pulmonary, colon and oral cancer, and leukaemia, and may contribute to the understanding of oncogenesis.

miRNAs may be a target for novel anticancer therapies. This will be the subject of our future research in the field of cervical cancer. 


\section{Acknowledgements}

The funds for this study were provided by a grant from the Romanian Research Ministry (41-030 PNCDI II Grant).

\section{References}

1. US Cancer Statistics Working Group: United States Cancer Statistics: 1999-2004 Incidence and mortality web-based report. US Department of Health and Human Services, Centers for Disease Control and Prevention and National Cancer Institute, 2007, Atlanta, www.cdc.gov/uscs. Accessed April 7, 2010.

2. Todorova I, Baban A, Alexandrova-Karamanova A and Bradley J: Inequalities in cervical cancer screening in Eastern Europe: perspectives from Bulgaria and Romania. Int J Public Health 54: 222-232, 2009.

3. Zur Hausen H: Viruses in human cancers. Science 254: $1167-1173,1991$

4. Li HP, Leu YW and Chang YS: Epigenetic changes in virus-associated human cancers. Cell Res 15: 262-271, 2005

5. Lee JO, Kwun HJ, Jung JK, Choi KH, Min DS and Jang KL: Hepatitis B virus X protein represses E-cadherin expression via activation of DNA methyltransferase 1. Oncogene 24: 6617-6625, 2005.

6. Esteller M: Epigenetics provides a new generation of oncogenes and tumour-suppressor genes. Br J Cancer 94: 179-183, 2006.

7. Guil S and Esteller M: DNA methylomes, histone codes and miRNAs: tying it all together. Int J Biochem Cell Biol 41: 87-95, 2009.

8. Faraoni I, Antonetti FR, Cardonea J and Bonmassar E: miR-155 gene: A typical multifunctional microRNA. Biochim Biophys Acta 1792: 497-505, 2009.

9. Jiang S, Zhang HW, Lu MH, He XH, Li Y, Gu H, Liu MF and Wang ED: MicroRNA-155 functions as an OncomiR in breast cancer by targeting the suppressor of cytokine signaling 1 gene. Cancer Res 70: 3119-3127, 2010.

10. Pierson J, Hostager B, Fan R and Vibhakar R: Regulation of cyclin dependent kinase 6 by microRNA 124 in medulloblastoma. J Neurooncol 90: 1-7, 2008.

11. Agirre X, Vilas-Zornoza A, Jiménez-Velasco A, et al: Epigenetic silencing of the tumor suppressor microRNA Hsa-miR-124a regulates CDK6 expression and confers a poor prognosis in acute lymphoblastic leukemia. Cancer Res 69: 4443-4453, 2009.

12. Kozaki K, Imoto I, Mogi S, Omura K and Inazawa J: Exploration of tumor-suppressive microRNAs silenced by DNA hypermethylation in oral cancer. Cancer Res 68: 2094-2105, 2008.

13. Toyota M, Suzuki H, Sasaki Y, Maruyama R, Imai K and Shinomura Y: Epigenetic silencing of microRNA-34b/c and $\mathrm{B}$-cell translocation gene 4 is associated with $\mathrm{CpG}$ island methylation in colorectal cancer. Cancer Res 68: 4123-4132, 2008.
14. Comey DC, Flesken-Nitkin A, Godwin AK, Wang W and Nikitin AY: MicroRNA-34b and MicroRNA-34c are targets of p53 and cooperate in control of cell proliferation and adhesionindependent growth. Cancer Res 67: 8433-8438, 2007.

15. Lujambio A, Calin GA, Villanueva A, Ropero $\mathrm{S}$, Sánchez-Céspedes M and Blanco D: A microRNA DNA methylation signature for human cancer metastasis. Proc Natl Acad Sci USA 105: 13556-13561, 2008

16. Iorio MV, Visone R, Di Leva G, Donati V, Petrocca F and Casalini P: MicroRNA signatures in human ovarian cancer. Cancer Res 67: 8699-707, 2007.

17. Wilting SM, van Boerdonk RA, Henken FE, Meijer CJ, Diosdado B, Meijer GA, Le Sage C, Agami R, Snijders PJ and Steenbergen RD: Methylation-mediated silencing and tumour suppressive function of hsa-miR-124 in cervical cancer. Mol Cancer 9: 167, 2010.

18. Fackler MJ, McVeigh M, Mehrotra J, Blum MA, Lange J, Lapides A, Garrett E, Argani P and Sukumar S: Quantitative multiplex methylation-specific PCR assay for the detection of promoter hypermethylation in multiple genes in breast cancer. Cancer Res 64: 4442-4452, 2004.

19. Weber B, Stresemann C, Brueckner B and Lyko F: Methylation of human microRNA genes in normal and neoplastic cells. Cell Cycle 6: 1001-1005, 2007

20. Saito Y, Liang G, Egger G, Friedman JM, Chuang JC, Coetzee GA and Jones PA: Specific activation of microRNA-127 with downregulation of the protooncogene BCL6 by chromatin-modifying drugs in human cancer cells. Cancer Cell 9: 435-443, 2006.

21. Lujambio A, Ropero S, Ballestar E et al: Genetic unmasking of an epigenetically silenced microRNA in human cancer cells. Cancer Res 67: 1424-1429, 2007.

22. Hackanson B, Bennett KL, Brena RM, Jiang J, Claus R, Chen SS, Blagitko-Dorfs N, Maharry K, Whitman SP, Schmittgen TD, Lübbert M, Marcucci G, Bloomfield CD and Plass C: Epigenetic modification of CCAAT/enhancer binding protein alpha expression in acute myeloid leukemia. Cancer Res 68: 3142-3151, 2008.

23. Yang Z, Chen S, Luan X, Li Y, Liu M, Li X, Liu T and Tang H: MicroRNA-214 is aberrantly expressed in cervical cancers and inhibits the growth of HeLa cells. IUBMB Life 61: 1075-1082, 2009.

24. Baohua L, Ying H, Feng Y, Yang L, Weiguo L and Xing X: Reduced miR-34a Expression in normal cervical tissues and cervical lesions with high-risk human papillomavirus infection. Int J Gynecol Cancer 20: 597-604, 2010.

25. Melar-New M and Laimins LA: Human papillomaviruses modulate expression of microRNA 203 upon epithelial differentiation to control levels of p63 proteins. J Virol 84: 5212-5221, 2010. 